Ivan Trapić

Robert Pezer

Jurica Sorić

https://doi.org/10.21278/TOF.42303

ISSN 1333-1124

eISSN 1849-1391

\title{
ATOMISTIC MODELLING OF 2D STRESS DISTRIBUTION AROUND DISCONTINUITIES
}

\begin{abstract}
Summary
Molecular dynamics simulations have been used for decades to investigate continuum mechanics failure to give the correct distribution of stress near discontinuities, such as holes and crack tips. In this paper, stress distribution around elliptical holes in a sheet material has been examined in an atomistic and a continuum model. Atomistic interactions are described by the Tersoff potential tuned for carbon. Calculations were conducted for the problem of stress distribution around the elliptic hole in a 2D graphene sheet subjected to the gradually increasing uniaxial tension load. The atomistic stress is calculated as spatial average utilizing Hardy's formulation. The results have been compared with the Kirsch solution for stress concentration at the edge of the circular hole. A quantitative measure for switching from atomistic to continuum model and vice versa has been proposed. Routes toward the effective data-driven coupling of macro- and micromechanical models where continuum mechanics approach fails are pointed out.
\end{abstract}

Keywords: $\quad$ molecular dynamics, stress, spatial averaging, multiscale modelling

\section{Introduction}

A unified understanding of continuum physical properties across scales from the nanoworld of atoms to millimetre-sized grain structures has been sought by scientists for some time now. Simultaneous development of sophisticated physical computational models and computer hardware has reached the point, where our ambitions to fully understand the emergence of various phenomena from scale to scale is within our grasp. The apparent simplicity of molecular dynamics (MD) equations hinders enormous difficulties in the unification of the phenomena description across the scales, where we are able to reach just several hundred nanometres in space and nanoseconds in simulation time. Those difficulties were addressed with several very successful methods of multiscale (MS) simulation approaches. The main idea is very simple and refers to the introduction of atomistic degrees of freedom only in a limited part of the model space, where continuum mechanics (CM) breaks down and fails to correctly describe the structural phenomena. Prominent examples of such MS phenomena are softening, strain and stress localization, yielding damage and material fracture. However, there are different ways to achieve MS coupling and up to now a single approach that can treat every possible problem of practical importance has not yet been devised. 
In this paper, we introduce quantitative criteria for the atomistic-to-continuum model coupling in a 2D graphene sheet system. Standard analytically solvable CM models of stress distribution around the elliptic hole are considered. The mechanical conditions comprise of an infinite 2D sheet, linear response material and the constant tensile load imposed far away from the center of the hole. The stress distribution obtained within the MD calculation using the realistic interaction potential and the convenient averaging procedure is analyzed and compared with the standard Kirsch analytical solution. The goal is to develop an efficient methodology to treat parts of the model space properly when the CM approach fails. However, this is far from an easy task. The essence of the problem in the MS modelling it comes down to two scale problems: time scale and spatial resolution. In the literature, there have been many interesting developments in the pursuit of an accurate and effective method to account for this and some of them that are relevant to the present study are briefly shown here.

In this paper, a review of the comparison between the atomistic MD model and the CM description in 2D structures is given. Presenting the advantages and disadvantages, we propose routes for effective utilization of the best features of both worlds: the atomistic approach for the phenomena that necessary involve discrete nature of the matter distribution including chemical bonding, and the efficient CM formalism, usually implemented within the finite elements method (FEM) with a myriad of fine-tuned tools developed through many years for the purpose of dealing with practical engineering problems.

\section{Multiscale modelling}

MS modelling establishes a material model that spans over several orders of magnitude in the time and length scales. Usually, MS methods are used to connect discreetness of atoms and the coarse nature of CM. By this coupling one is able to study the nanostructure of the localized region in the same model. Of special importance are parts of the model space where dynamics of individual atoms is relevant, which is appropriate for the MD approach. Otherwise, we are free to use computationally less expensive CM, in which deformation is homogenous and smooth enough. Two branches of the MS development have come into existence to address the issue: hierarchical and concurrent methods.

The hierarchical methods implement sequential calculations on the atomistic and the continuum level. Simulations are completely separated and the only necessary information is exchanged between the models, usually as boundary conditions. The analysis is first carried out on a representative volume element, which is equipped with microscopic information from the atomistic calculation giving us more accurate physically based constitutive relations for the continuum level.

In the concurrent methods, the model space is divided into an atomistic and a continuum region. By using the matching procedure in the part of the system described simultaneously by both continuum and atomistic degrees of freedom the description is improved combining different scales and exchanging relevant physics information. In this case, two or more different scales are present simultaneously in the same model. In this approach, the atomistic model that is studied by using MD is usually surrounded by a finite element (FE) mesh. Some of the first results in the atomistic/FEM combination where obtained by Kohlhoff et al. [1]. Other very popular approaches are the Quasicontinuum (QC) method developed by Tadmor et al. [2] and Bridging Domain (BD) developed by Belytschko et al. [3]. Very important contribution to the atomistic part of the MS approach is a combination of the MD techniques (introduced in [4]) with realistic potentials, such as the Daw and Baskes embedded atom method [5].

In the QC method the coupling of the atomistic and the continuum model is achieved by refining the FE mesh in the "troubled" region until one node corresponds to one atom. In the rest of the model, one FE usually contains many atoms. In the regions described by the 
atomistic approach the motion of nodes/atoms is determined from MD equations. In the regions of the continuum approach, where one FE represents a set of atoms, it is not necessary to track the displacement of each and every individual atom. Instead, it is sufficient to analyze only several atoms coincident with FE nodes. The QC method has been used to study basic properties of deformation in the crystalline solid, fracture and grain boundary slip.

To make the transition from MD to CM less sudden, in the BD method the so-called handshake region (HR) is established, in which atomistic and continuum models meet. Compatibility between the atomistic and the continuum method is achieved by imposing an interpolated displacement field on the HR atoms. To avoid double energy counting in the HR, the total energy calculation as a sum of atomistic and continuum energies has to be performed with special care. The BD method is suitable for studying nanodefects on a higher scale, but the region of interest has to be far enough from the HR.

Fig. 1 shows the basic principle of the domain partition in the BD and the QC approach. The atomistic domain $\Omega_{\mathrm{a}}$ is analyzed by MD, while the discretization of the continuum part $\Omega_{\mathrm{c}}$ is done by using the FEM. In the case of $\mathrm{BD}$, both models are present and overlap in the HR $\Omega_{\mathrm{b}}$.
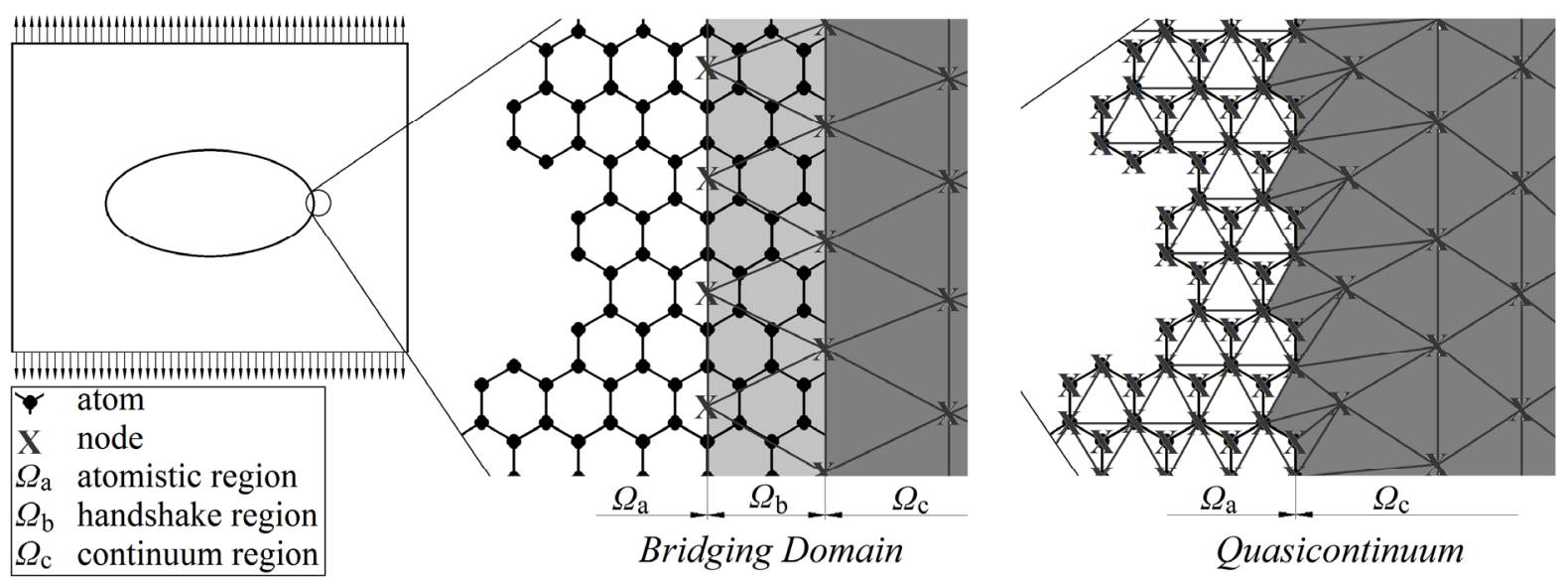

Fig. 1 Type of transition between atomistic and continuum models in different multiscale methods

\section{Molecular dynamics}

The strategic approach of MD is to explicitly consider every individual atom whenever continuum based methods, such as FEM, are not capable of capturing phenomena that are discrete at the fundamental level. An MD system is essentially one where all the atomic nuclei in the system are treated as classical Newtonian particles without internal degrees of freedom and all quantum many-body effects are phenomenologically imprinted in the interaction potential.

If one wishes to study the system at the temperature of absolute zero it is sufficient to set atom velocities to vanish. By finding local energy minima of the given atomistic configuration one can study the energy landscape and transition paths between different meta stable states. Since the potential energy surface is very complicated, finding the energy minimum demands highly convex iterative numerical procedures, such as steepest descent, conjugate gradient or the Newton-Rapson method. Unfortunately, there is no guarantee that the found local minimum is the global minimum, so repeating the simulation and scanning other parts of the energy landscape is usually necessary.

In systems at a finite temperature the velocities are nonzero and therefore atoms are constantly in motion. Classical Newton equations of motion can be numerically integrated by the Verlet algorithm [6]. Usually, the half-step velocity formulation of the algorithm is 
implemented, which saves memory consumption since there is no need to save values from the previous time step. This algorithm is simple, stable, energy conservative and symplectic. However, finding the right value for a time step can be somewhat tricky since a too small time step would unnecessarily prolong the calculation time and a too large time step would cause the unphysical behavior of the system. When full-time evolution of the system is performed thus providing trajectories of the atoms we get a direct insight into the mechanics of material deformation under loading. The main problem of connecting properties of particles such as trajectories, velocities and interatomic forces from an MD simulation with a stress field within the CM framework is in the ambiguities since there are many ways to connect atomistic and continuum properties.

In the atomistic simulation, the standard numerical infrastructure of LAMMPS (stands for Large-scale Atomic/Molecular Massively Parallel Simulator and provides a de facto standard in robust MD equations integration) has been used [7]. LAMMPS is a classical MD simulation code that models an ensemble of particles in various matter states including a high level of flexibility in terms of extending and adapting the system for a specific task.

\subsection{Interaction potential}

Forces between atoms are derived from empirical interatomic potentials that are obtained by adjusting free parameters to material properties (e.g., lattice constant, elastic constants, vacancy-formation energy, etc.) from experimental data or ab initio quantum mechanics calculations. In the case of graphene, we use the very well established Tersoff interatomic potential for carbon from [8]. Physicist J. Tersoff developed a convenient approach to effective interactions by first abandoning the traditional many-body route and proposing a simple idea that the strength of pair potential depends upon the local atomic environment in such a way that an increasing number of neighbours weakens the bonds. In this way he was able to develop highly successful models first for silicon and then for carbon. The model that we use here proves to be very good for an accurate description of migration barriers, elastic constants and phonon frequencies.

Theoretically, the interaction between the atoms is infinite in range, however, the screening effect of the interaction in many-body systems usually causes finite range interactions among neutral atoms. Therefore, after certain distance the contribution of an atom that is further away is negligible compared to the surrounding atoms. This fact can be utilized for the computation time reduction without loss of accuracy. Only interactions with atoms within certain cutoff radius $r_{\text {cut }}$ (shown in Fig. 2) are included and all other interactions are discarded.

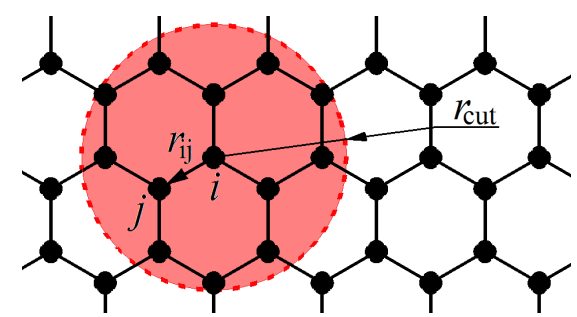

Fig. 2 Graphic representation of the cutoff radius

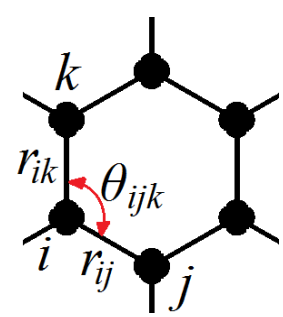

Fig. 3 Bond angle between atoms

The used potential contains two- and three-body interactions which determine properties and behavior during deformation loading. Three-body interactions are essential for a quantitative model of the structural graphene properties such as the angle of $120^{\circ}$ between the bonds on the tips of the hexagonal lattice structure. The total potential energy $E_{\mathrm{p}}$ of the system with $N$ atoms is the sum of interactions as shown in equations (1)-(5). $V_{\text {ij }}$ is the potential energy of pairwise interactions of atoms $i$ and $j$, where $r_{i j}=r_{i}-r_{j}$ is the distance between 
them. Three-body interactions are implemented in the potential by function $z_{i j}$ which contains angle $\theta_{i j k}$ between vectors $r_{i j}$ and $r_{i k}$ as shown in Fig. 3. A more detailed description of the potential can be found in [9] and the parameter values for the carbon system in [8].

$$
\begin{aligned}
& E_{\mathrm{p}}=\sum_{i=1}^{N} E_{i}=0.5 \cdot \sum_{i=1}^{N} \sum_{\substack{j=1 \\
j \neq i}}^{N} V_{i j} \\
& V_{i j}\left(r_{i j}\right)=f_{c}\left(r_{i j}\right) \cdot\left[A \exp \left(-\lambda_{1} r_{i j}\right)-B_{i j} \exp \left(-\lambda_{2} r_{i j}\right)\right] \\
& B_{i j}=B_{0} \exp \left(-z_{i j} / b\right) \\
& z_{i j}=\sum_{i=1}^{N} \sum_{\substack{j=1 \\
j \neq i}}^{N} \sum_{\substack{k=1 \\
k \neq j}}^{N}\left[w\left(r_{i k}\right) / w\left(r_{i j}\right)\right]^{n} \times\left[c+\exp \left(-d \cos \theta_{i j k}\right)\right] \\
& w\left(r_{i j}\right)=f_{c}\left(r_{i j}\right) \exp \left(-\lambda_{2} r_{i j}\right)
\end{aligned}
$$

\subsection{Ensemble theory}

Many-body problems are ubiquitous in describing natural phenomena. On the other hand, they present one of the most difficult challenges to the scientific community. One very successful answer to these questions is the statistical physics approach. The number of atoms in MD simulations is anywhere in the range of several million to even billions but nonetheless, that is an almost unthinkably small number in comparison with macroscopic systems since one gram of carbon contains an order of $10^{23}$ atoms. The sheer magnitude of this number makes any attempt to describe a macroscopic system in terms of mechanical properties of its microscopic constituents extremely difficult. At the heart of the usefulness of the MD simulation is the framework of ensembles - "imaginary copies" of the system under the same physical constraints. In accordance with an ergodic hypothesis, the main idea is to replace ensemble averages with time averages from the MD simulation for the calculation of thermodynamic observables. Common textbook ensembles in statistical physics are microcanonical (NVE), canonical (NVT) and isothermal-isobaric (NpT) ensembles. Here we calculate equilibrium properties during loading of the sheet of material in the $\mathrm{NpT}$ ensemble since this one usually corresponds to the experimental conditions for condensed matter.

\subsection{The problem of stress at an atomistic level}

The subject of interpreting highly successful CM models in terms of atomistic degrees of freedom proves to be a formidable task that started with the pioneering work of J. C. Maxwell [10], followed by Irvin and Kirkwood [11], Hardy [12] and Tsai [13]. It is important to state that the subject is still an active area of research [14], [15], [16], [17]. Basic knowledge of the subject is given in the papers of famous physicists J.C Maxwell [10] and R. Clausius. Their results came simultaneously with the development of statistical mechanics, where they successfully developed a connection between virial expansion and continuum stress formulation besides other physical quantities. It is not only important to give a proper explanation of statistical averaging that leads to $\mathrm{CM}$ but also to inform macroscale models in very important macrophenomena that are governed by atomistic scale physics. The examples are plastic deformation and failure of the material that, as is shown, operate at a full range of scales. The advent of modern computers motivated Irvin and Kirkwood to formulate the first practical models of stress calculation in terms of underlying atomistic degrees of freedom [11] (equation (6)). Further development is presented in the paper by Hardy [12], who 
quantitatively introduced the fact that atoms occupy volumes with some space distributions and this approach is applicable for inhomogeneous systems as presented in the expression for the Cauchy stress tensor of the atomistic model (equation (7)).

$$
\begin{aligned}
& \boldsymbol{\sigma}(\mathbf{r})=\frac{-1}{\Omega(\mathbf{r})}\left(\sum_{\alpha=1}^{N} m^{\alpha} \mathbf{v}^{\alpha} \otimes \mathbf{v}^{\alpha}+\frac{1}{2 !} \sum_{\substack{\alpha=1 \\
\alpha}}^{N} \sum_{\substack{\beta=1 \\
\beta \neq \alpha}}^{N} \mathbf{f}^{\alpha \beta} \otimes \mathbf{r}^{\alpha \beta}+\frac{1}{3 !} \sum_{\substack{\alpha=1 \\
\beta=1 \\
\beta \neq \alpha}}^{N} \sum_{\substack{\gamma \neq 1 \\
\gamma \neq \beta}}^{N} \mathbf{f}^{\alpha \beta \gamma} \otimes\left(\mathbf{r}^{\alpha \beta}+\mathbf{r}^{\alpha \gamma}\right)+\ldots\right) \\
& \tilde{\boldsymbol{\sigma}}(\mathbf{r})=-\left[\frac{1}{2} \sum_{\alpha=1}^{N} \sum_{\beta=1}^{N} \mathbf{r}^{\alpha \beta} \otimes \mathbf{f}^{\alpha \beta} B^{\alpha \beta}(\mathbf{r})+\sum_{\alpha=1}^{N} m^{\alpha} \hat{\mathbf{v}}^{\alpha} \otimes \hat{\mathbf{v}}^{\alpha} \psi\left(\mathbf{r}^{\alpha}-\mathbf{r}\right)\right]
\end{aligned}
$$

Here Hardy introduces $\psi$ and $B$ distribution functions normalized to unity that effectively takes the fact that atoms (electron cloud) overlap in solid state systems into consideration. The approach of Hardy is similar to the Irving and Kirkwood procedure, but formal expressions are more naturally implemented in MD simulations.

\subsection{Stress averaging procedure}

For calculating stress value in an arbitrary position of space the first step is to choose an appropriate averaging radius which determines the maximum distance of atoms that contribute to the stress value at a particular position as shown in Fig. 4. Choosing an averaging radius is not completely arbitrary since a too small value would result in a meaningless per atom stress value and a too large averaging radius value would decrease the relevant atomistic contribution spoiling the spatial resolution so that possibly important local complex stress variations will be smeared out to such an extent that they are no longer visible.

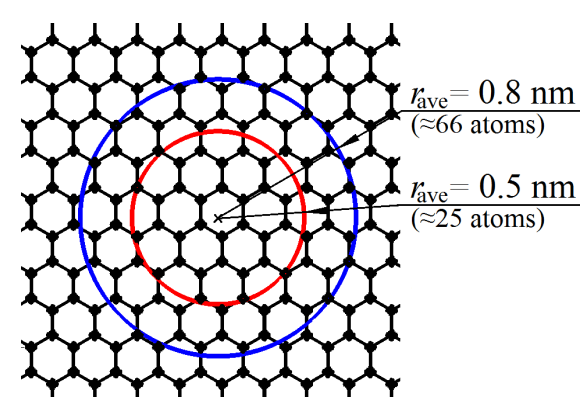

Fig. 4 Determination of the averaging radius (all atoms within the circle are taken into account for the stress averaging procedure)

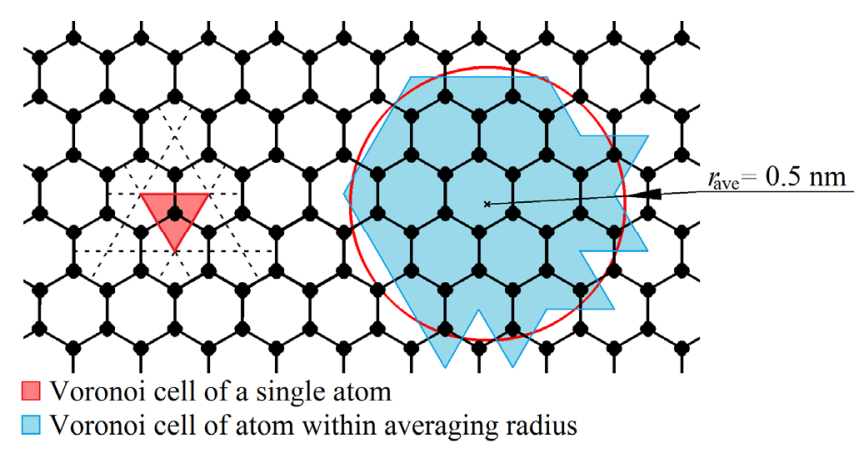

Fig. 5 Representation of the Voronoi tessellation used for volume per atom determination

Upon choosing the optimal value of the averaging radius, the procedure goes as follows:

1. define a representative set of points in space to conveniently calculate the stress distribution

2. choose one point in space and identify all atoms within the averaging radius around that point

3. add up all stress contributions per chosen atoms with the following equation (this is a variant of expression (6) only without the volume $\Omega$ )

$$
\tilde{\sigma}_{i j}=-\left[m v_{i} v_{j}+\frac{1}{2} \sum_{\kappa=1}^{N_{p}}\left(r_{i}^{\kappa, 1} f_{j}^{\kappa, 1}+r_{i}^{\kappa, 2} f_{j}^{\kappa, 2}\right)+\frac{1}{3} \sum_{\zeta=1}^{N_{a}}\left(r_{i}^{\zeta, 1} f_{j}^{\zeta, 1}+r_{i}^{\zeta, 2} f_{j}^{\zeta, 2}+r_{i}^{\zeta, 3} f_{j}^{\zeta, 3}\right)+\ldots\right]
$$

The tilde above the stress symbol points out that the measurement for this stress is in Nm since it is not divided by the volume. The second term is the contribution of the pairwise interactions where $\kappa$ loops over $N_{\mathrm{p}}$ neighbours of the atom within the cut off radius, $\mathbf{r}^{\kappa, 1}$ and 
$\mathbf{r}^{k, 2}$ are the positions of the atoms in pair interactions and $\mathbf{f}^{\kappa, 1}$ and $\mathbf{f}^{k, 2}$ are the interaction forces. In a similar way, $N_{\mathrm{a}}$ is the number of angle-dependent interaction contributions, where $\mathbf{r}$ and $\mathbf{f}$ are atom positions and interaction forces, respectively.

4. divide thus the obtained cumulative stress with the total volume (area in our 2D case) summing the volumes per identified atoms to give the final stress distribution expression as follows:

$$
\boldsymbol{\sigma}(\mathbf{r})=\frac{\sum_{\alpha=1}^{N} \tilde{\boldsymbol{\sigma}}^{\alpha}}{\sum_{\alpha=1}^{N} \Omega^{\alpha}}
$$

Herein $N$ is the number of the atoms within the volume, $\Omega^{\alpha}$ is the volume obtained by partitioning the space utilizing the Voronoi tessellation procedure and $\boldsymbol{\sigma}^{\alpha}$ stress of the atom. Fig. 5 shows the results of the Voronoi tessellation for the ideal graphene structure for one atom and all atoms within the averaging radius.

The stress distribution of the material with holes of elliptical shape is described using the proposed method within the framework of the combined MD and CM description. The values of the free parameters in the implementation have to be adjusted in the regions far from the hole where both models give an essentially identical result. In this way we are able to obtain limits of the CM approach since the scanning of the model space identifies regions of a significant discrepancy between the $\mathrm{CM}$ and the MD results.

\section{Numerical computation}

\subsection{Simulation model}

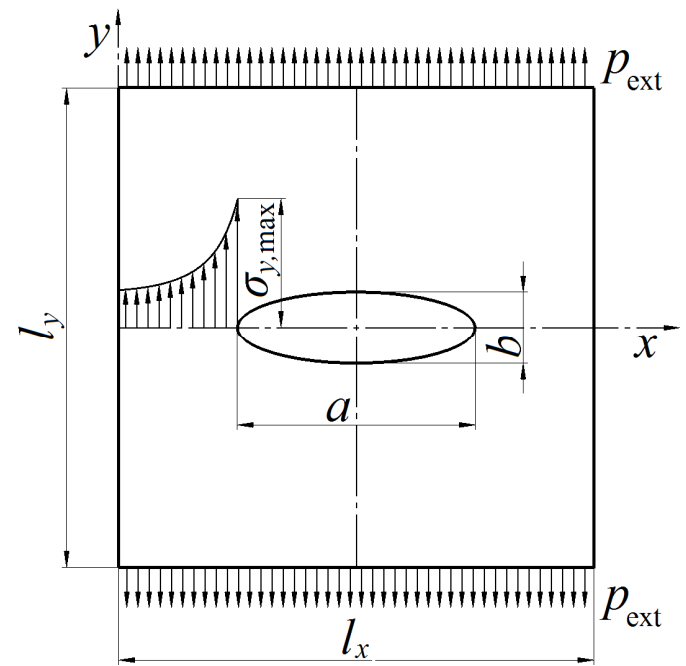

Fig. 6 Geometry of the 2D plate with an elliptic hole subjected to tension and stress distribution along the $x$-axis

A strategic approach to investigate the continuum/atomistic discrete correspondence is chosen to be simple, but on the other hand, large enough to provide clear signatures of critical features signaling a CM failure. The system of carbon atoms arranged in a graphene 2D honeycomb structure with an elliptic hole in the middle has been prepared with common realistic features provided by the interatomic potential. Fig. 6 shows the system with characteristic features, load and stress distribution. Table 1 contains values of the model properties used in the numerical calculation. Initial thermalization of the system was done at 
the desired temperatures: at 10 and $300 \mathrm{~K}$ for $20 \mathrm{ps}$. After that, the graphene sheet was pulled in the $y$-direction by simultaneously imposing the $\mathrm{NpT}$ ensemble conditions with a finite value of temperature and pressure at the edges of the simulation cell for a sufficient length of time so that full system thermalization is achieved. Calculations were performed for two temperatures: 10 and $300 \mathrm{~K}$ by coupling the system to the standard Nosé-Hoover thermostat [18], [19] ensuring canonical distributions during the simulation.

Table 1 Properties of the atomistic model

\begin{tabular}{|c|l|}
\hline Sheet size (before loading) & $\begin{array}{l}l_{x}=174 \mathrm{~nm} \\
l_{y}=172 \mathrm{~nm}\end{array}$ \\
\hline ellipse size and proportion & $\begin{array}{l}a=34,26,18,10,2 \mathrm{~nm} \\
a / b=1,2,30\end{array}$ \\
\hline temperature & $T=10,300 \mathrm{~K}$ \\
\hline initial thermalization time & $t_{\text {therm }}=20 \mathrm{ps}$ \\
\hline external load & $p_{\text {ext }}=150 \mathrm{GPa}$ \\
\hline loading time & $t_{\text {load }}=100 \mathrm{ps}$ \\
\hline number of atoms & $N_{\text {atoms }} \approx 1.1 \mathrm{million}$ (depends on the hole dimensions) \\
\hline averaging radius & $r_{\text {ave }}=1.8 \mathrm{~nm}$ \\
\hline average number of atoms within an average radius & $N_{\text {ave }} \approx 287$ \\
\hline
\end{tabular}

\subsection{Results}

As a didactic example, we prepared a simple model with only 1984 atoms so that the movement of individual atoms can be followed more easily. Fig. 7 shows the initial unloaded configuration. Please note that the hole is only approximately elliptical since the discrete nature of the atomistic system gives jagged edges with unsymmetrical atom bonds.

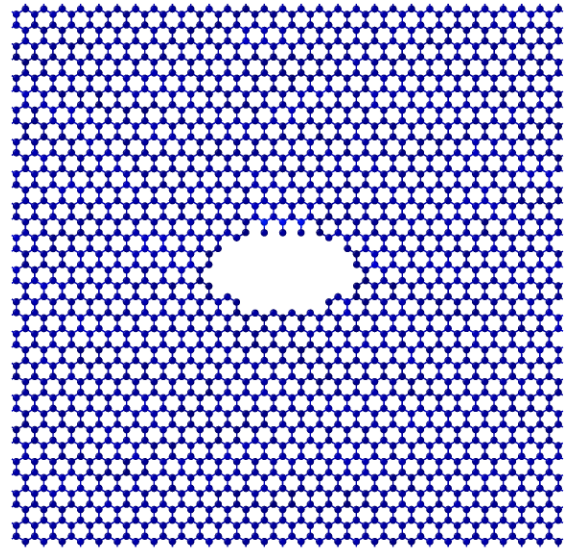

Fig. 7 Initial unloaded configuration

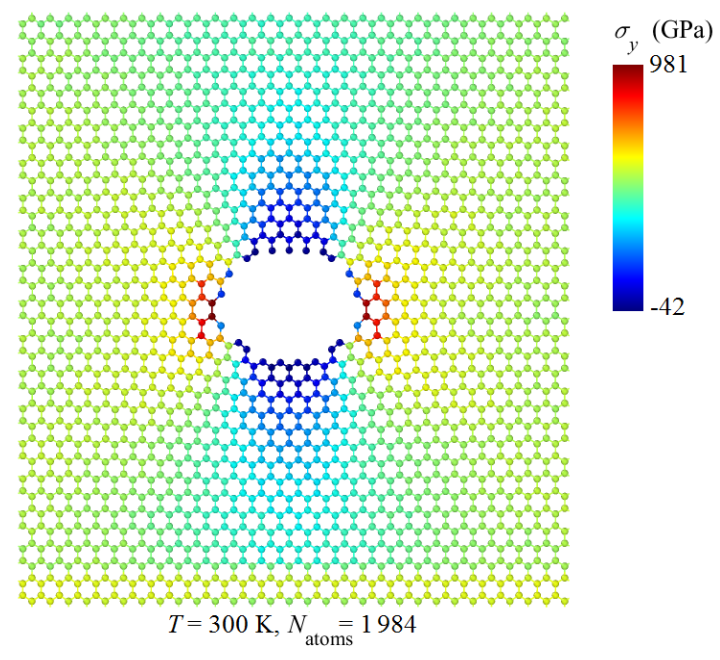

Fig. 8 Per atom $\sigma_{y}$ stress distribution on the deformed configuration at a temperature of $300 \mathrm{~K}$

This is the region where the most pronounced discrepancy between the continuum and the atomistic model results is to be expected due to the boundary effects which play a significant role in the graphene properties as shown in [20]. Fig. 8 shows the per atom stress distribution with a clear indication that the edge atoms are weakly bounded. Figs. 7 and 8 were generated by using OVITO [21] (Open Visualization Tool). From the figures, it is clear that in this case there is a narrow region around the hole where the CM results break down at the atomic scale since they are unable to catch the discrete nature of atomic bonding. This will have a major impact in the presence of nanoscale defects in graphene. Particularly important is the realistic 
case where defects can be distributed in a myriad of ways and have a significant impact on mechanical properties. Since the sheet of graphene can be treated as an isotropic material when subjected to the in-plane loading, as suggested in [22], a suitable analytical solution for comparison is the Kirsch solution [23]. The most prominent feature of the solution is an increase in the stress from the remote loading value $\sigma_{\infty}$ to the three times higher peak value at the edge of the hole as shown in Fig. $6\left(\sigma_{y, \max }=3 \sigma_{\infty}\right)$. The convenience of this model is the existence of an analytic solution and nontrivial stress distribution with interesting edge features that are very different as we go down to the atomistic scale of atomic bonding.

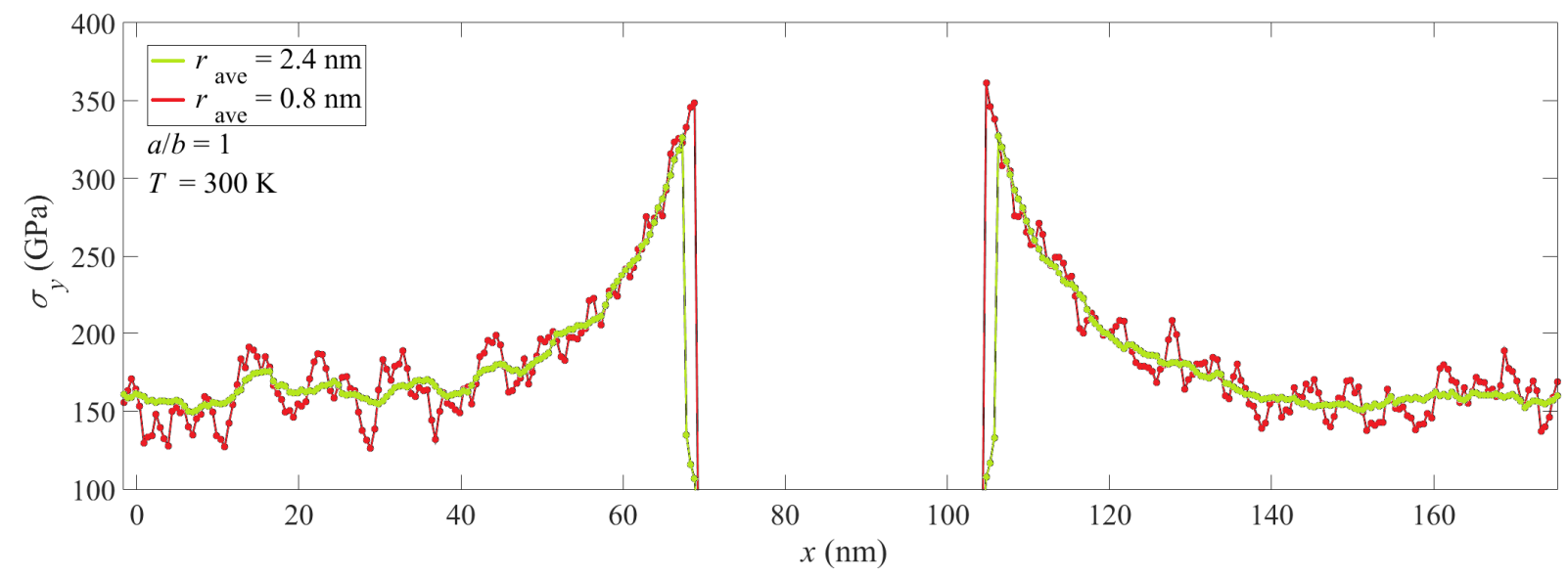

Fig. 9 Comparison of $\sigma_{y}$ stress distribution for different averaging radii

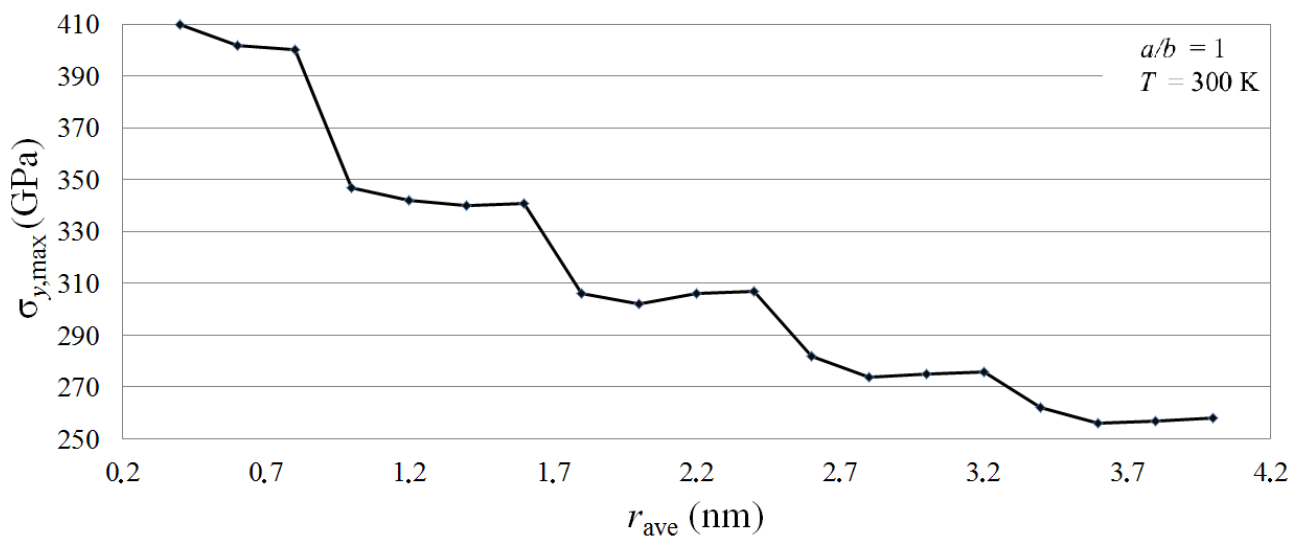

Fig. 10 Maximal $\sigma_{y}$ stress at the hole edge for different averaging radii

All calculations of the spatially averaged stress were performed at a temperature of $300 \mathrm{~K}$. In Fig., the results of the $\sigma_{y}$ stress calculation along the $x$-axis for different averaging radii are shown. The unsymmetrical values of stresses on the left-hand and right-hand side of the hole despite the symmetrical loading and geometry is a consequence of the unsymmetrical atom bonds around the hole as it can be seen in Fig. 7. In addition to the obvious smearing out of the fluctuations, the graph shows a systematic reduction in the peak stress value that is shown in Fig. 10 with a visible step like a drop effect approximately every $0.5 \mathrm{~nm}$ (experimental nearest neighbour distance of carbon atoms in graphene is $0.142 \mathrm{~nm}$ ). The origin of this effect is a discrete jump in the number of atoms included in the calculation as we increase the averaging circle radius. The maximum stress drop is less and less pronounced since the fraction of the new bunch of atoms that join the circle is smaller and smaller. The averaging and determination of a proper statistical procedure is actually situation dependent. The main obstacle in coupling different models is visible from the characteristic length and time scales as it is usually a fact in MD simulations. 
Let us illustrate the problem by comparing different length scales in the case of simple uniaxial loading. For a realistic system at the atomic scales, a typical vibration period is around fs $\left(10^{-15} \mathrm{~s}\right)$, so in order to utilize the standard computational MD method of Alder and Wainwright [4], one is forced to impose strain rates of the order at least $10^{6} \mathrm{~s}^{-1}$ in comparison to the typical experimental values $10^{-4} \mathrm{~s}^{-1}$. So the standard mechanical test strain rate is ten orders of magnitude separated from what is computationally achievable in the MD simulation. The point is that the MD simulation is localized on a very tiny part of the experimental specimen so the information about the applied tension is not uniform across the volume at the atomistic time scale. From the atomistic perspective, the tensile test can be considered as a quasi-static process for a myriad connected atomistic systems and one of the routes to connect the two worlds is hybrid modelling [24].

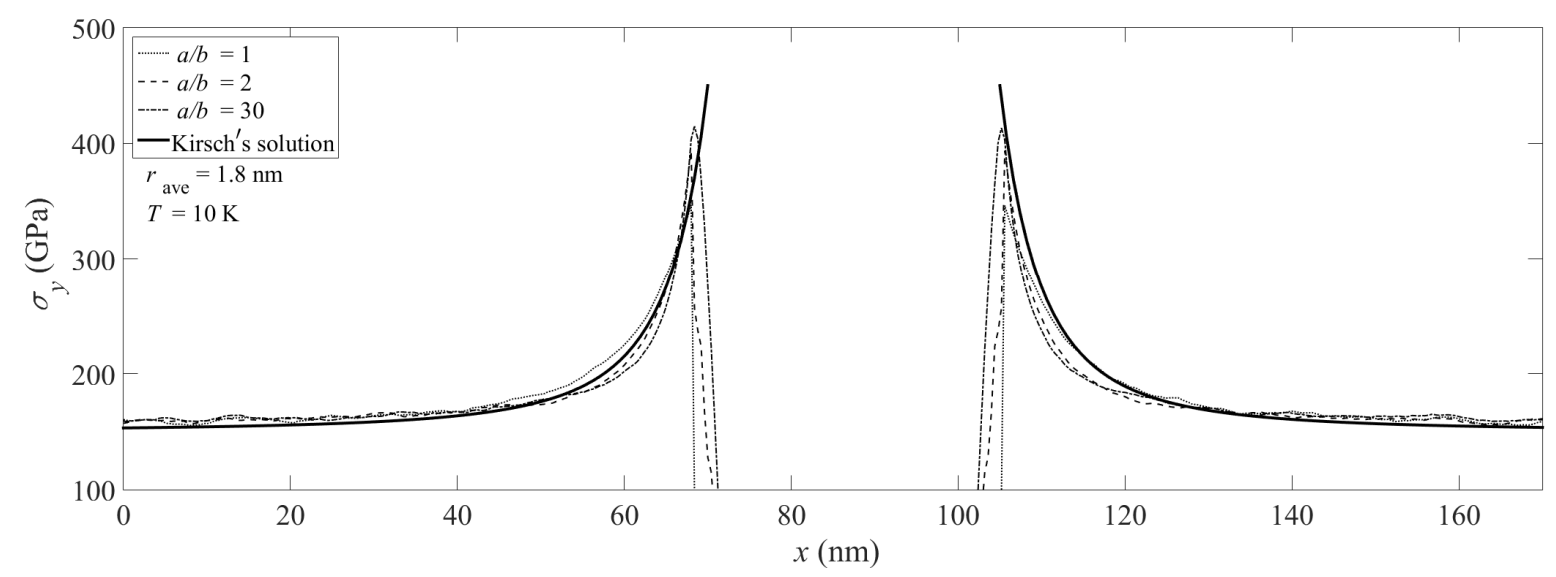

Fig. 11 Normal stress $\sigma_{y}$ distribution along the $x$-axis for different ratios of the ellipse dimensions compared to an exact analytical solution in the continuous limit for $a=34 \mathrm{~nm}$ at temperature of $10 \mathrm{~K}$

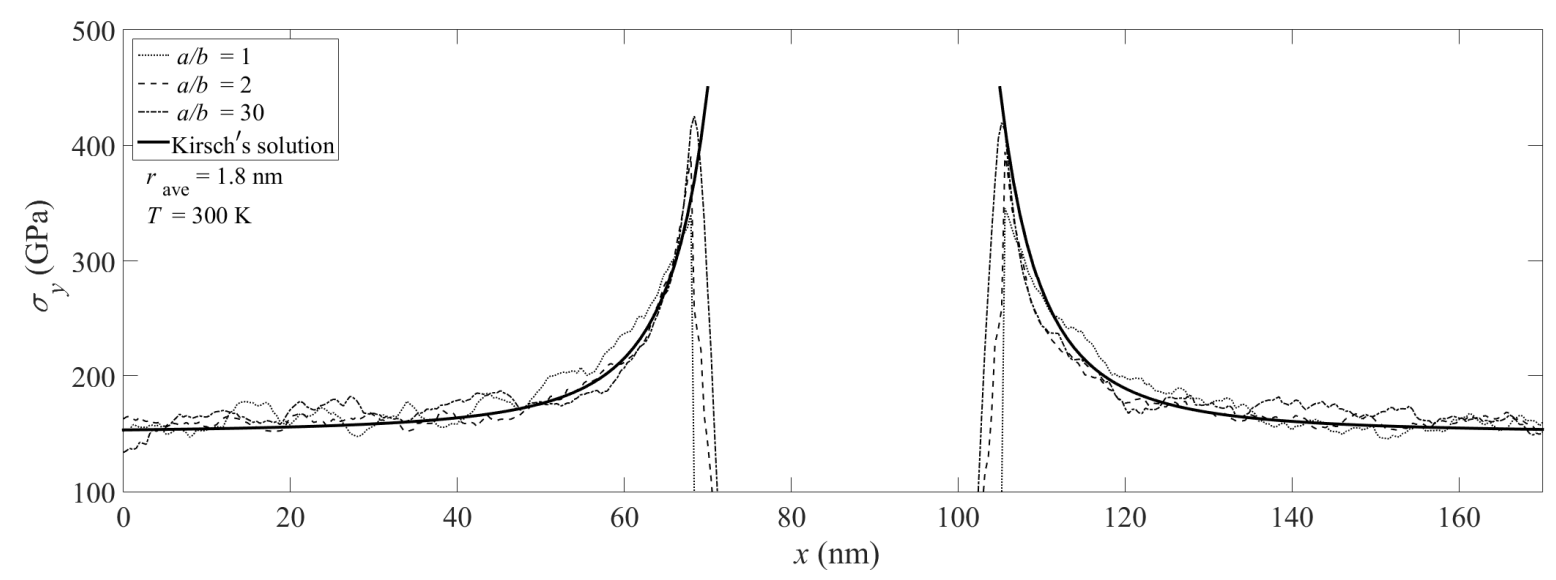

Fig. 12 Normal stress $\sigma_{y}$ distribution along the $x$-axis for different ratios of the ellipse dimensions compared to an exact analytical solution in the continuous limit for $a=34 \mathrm{~nm}$ at temperature of $300 \mathrm{~K}$

Here we have chosen a different route and examined a simple averaging procedure but with a variable averaging circle diameter with no temporal averaging. In this way we present results at a specific microscopic moment. In the end, we would like to develop more elaborate approaches, so here we are trying to be as simple as possible keeping relevant information. Fig. 11 shows the $\sigma_{y}$ stress along the $x$-axis for different elliptical eccentricities. It is interesting to note that the thermal signature on the trend is the increase in fluctuations. So for the same averaging radius the fluctuations are much more pronounced for the loading at 300 $\mathrm{K}$ than for the loading at $10 \mathrm{~K}$. A relatively small number of particles in the averaging circle is the reason why the time fluctuations are visible at a higher temperature (only spatially 
averaged stress). As can be seen from Figs. 11 and 12, the atomistic and the continuous model show remarkable overall agreement of the stress distribution in the Kirsch problem. Similar results have been obtained for other values of parameter $a$ with the only difference that a smaller hole results in a lower maximal stress. This shows that the stress distribution around the hole is mainly a topological feature in which the hole size determines only the peak value.

If it is taken into consideration that the Tersoff realistic interatomic potential is capable of describing very important microscopic mechanical properties, such as phonon dispersion curves [25] and elastic properties [26] (for experimental values see for example [27]), we are inspired to look for an effective model to join the continuum and the atomistic-level approach. We propose that void-like structures in realistic 2D materials can be concurrently modelled by using CM formalism away from void edges and the MD simulation within the properly chosen region close to the hole edges. As can be seen from Figs. 11 and 12, the continuum model overestimates stress at the very edge of the hole. This is caused by artificial propagation of the continuum picture of matter down to the atomistic scale, failing miserably to capture the reality that shows weakly bonded atoms with low-stress values. In the real atomistic system, the stress is at its maximum value slightly in the interior of the material, giving precise information where the stress concentration is critical.

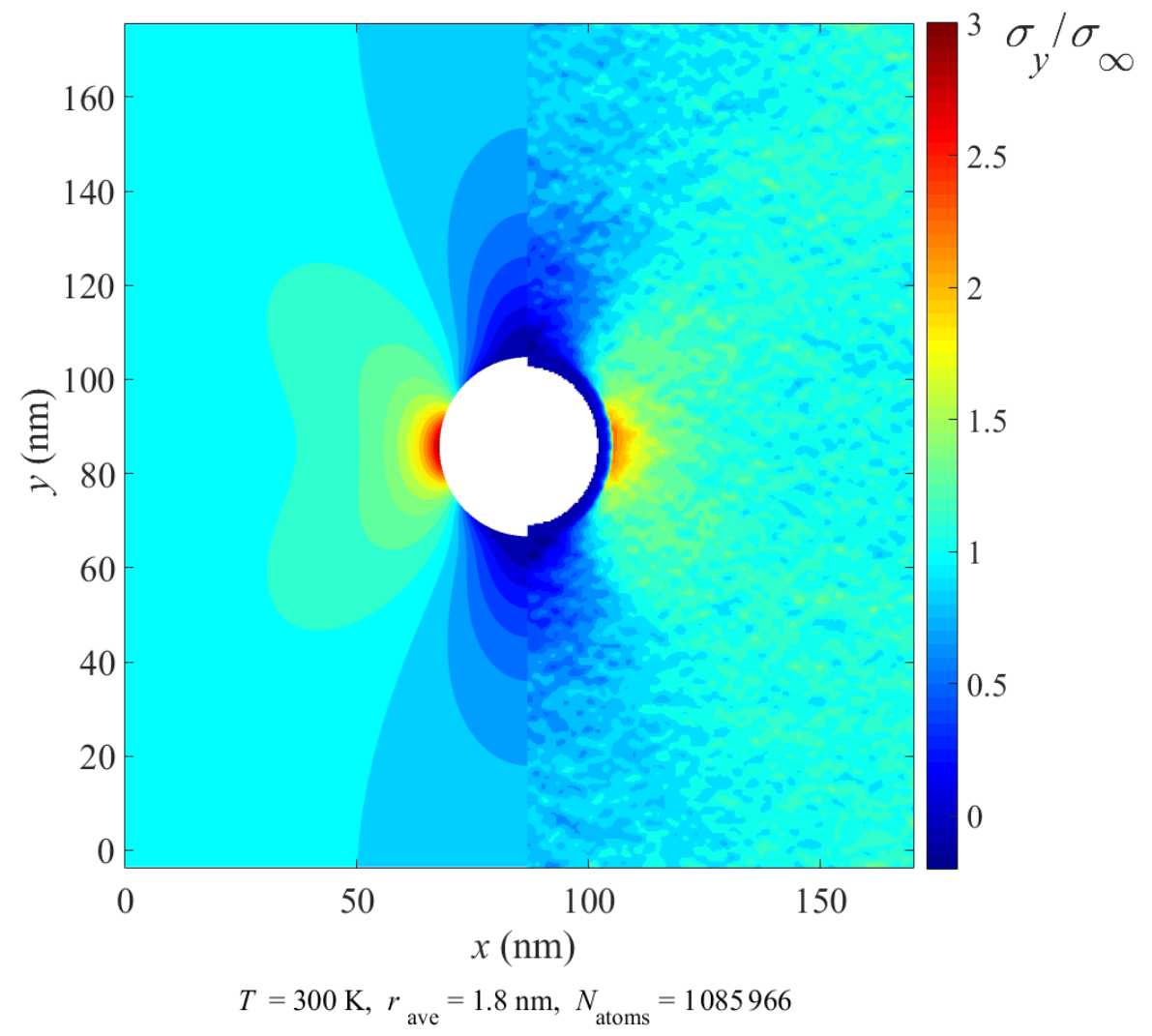

Fig. 13 Comparison of the continuum (left-hand part of the model) and spatially averaged stress atomistic stress distribution (right-hand part of the model)

In Fig. 13 we show a comparison of the $\sigma_{y}$ stress distribution over the whole model space calculated by spatial atomistic averaging and the analytical $\mathrm{CM}$ results. The space averaging circle radius is chosen to indicate inhomogeneity of the averaged atomistic stress. Since the problem is symmetric, the left half of Fig. 13 presents the CM while the right part presents the atomistic results. Motivated by this correspondence we propose a quantitative measure identifying the region where one should switch from CM to the atomistic description and this is the ratio of the atomistic to the continuum stress around a hole. 


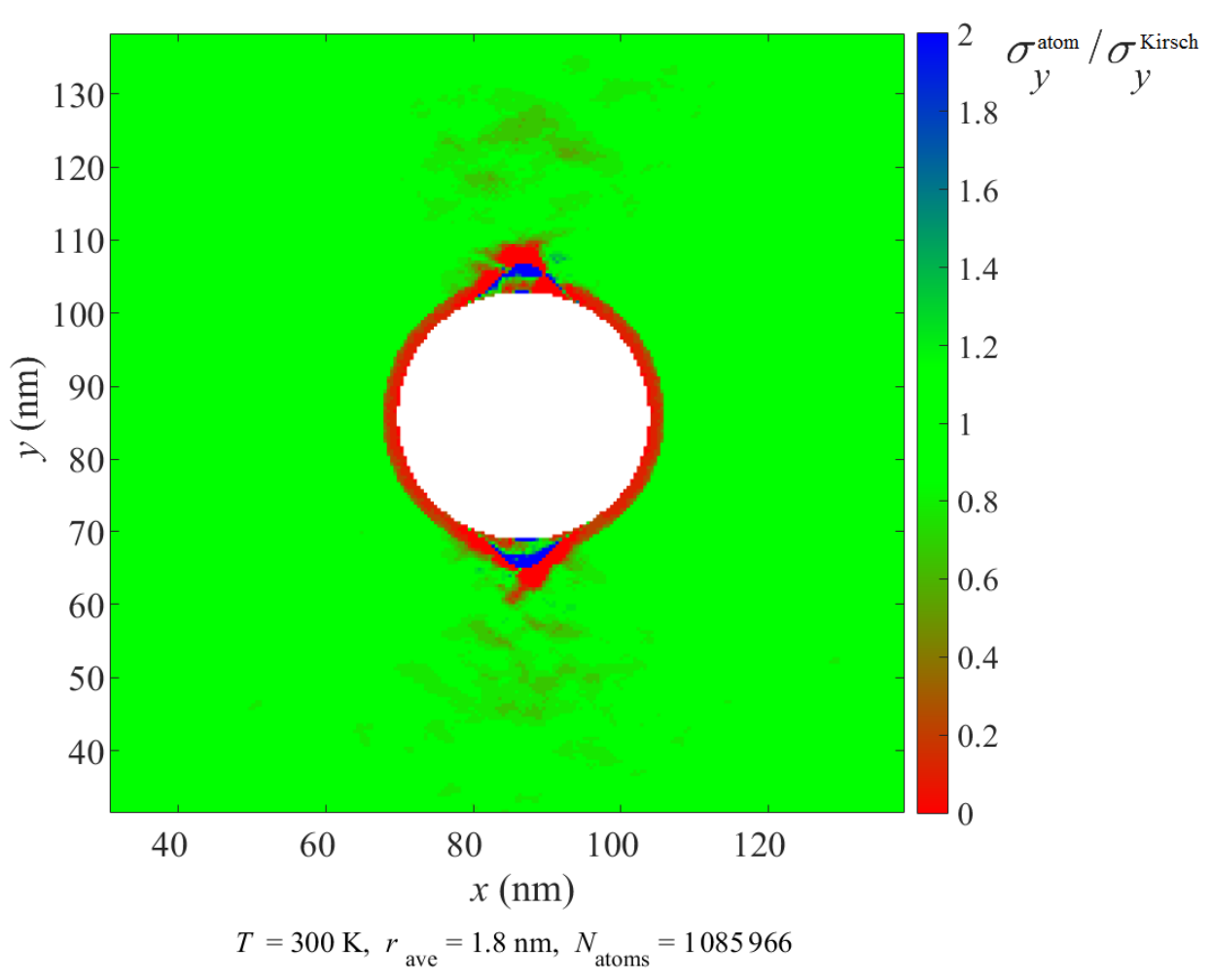

Fig. 14 Spatial averaged atomistic to continuum stress ratio distribution around the hole quantitatively indicating discrepancy of the results

We propose the following quantitative criteria that can be conveniently adjusted to suit the practical problem at hand:

$$
\begin{array}{ll}
1-\alpha<\sigma_{\mathrm{a}} / \sigma_{\mathrm{c}}<1+\alpha & \text { continuum mechanics } \\
1-2 \alpha<\sigma_{\mathrm{a}} / \sigma_{\mathrm{c}}<1+2 \alpha & \text { combined (e.g. handshaking region) } \\
\text { rest } & \text { atomistic }
\end{array}
$$

where $\alpha$ is some small positive constant estimated close to 0.15 . This threshold parameter can be further optimized depending on the problem complexity and corresponding computational cost. The main aim of the MS approach is to solve complex mechanics problems and in this case it comes down to judiciously reconcile physical quantities at the atomistic level that carry out vast quantities of information to macroscopic quantities, such as stress, by using a suitable averaging procedure. The main routes are given by standard statistical physics theories but the problem is that they are usually too general and need fine tuning to a specific problem in materials engineering. Switching from coarser to finer algorithms and keeping the necessary physical information in the model is a rather subtle subject. This is because matching such a different models that disagree even upon the basic structure of matter proves to be challenging [16]. In Fig. 14 we show how we can easily identify regions of the sheet where the atomistic and the CM stress distribution do not match. Fortunately, the regions are clearly localized indicating that vast portions of the model space can be described within the CM framework. Once the regions are clearly and effectively identified we can choose some of the standard atomistic to CM coupling approaches [3]. One of the problems to be solved is the topology of the system identification and geometry analysis that has to be effective enough to give the computational and physical description advantage. Motivated by the previously shown results we propose the following two-step procedure:

1. topology scanning - holes identification and atomistic region definition

2. self-consistent calculation of the stress distribution within the $\mathrm{CM}$ and the atomistic regions of the model space 
Here we have chosen the self-consistent calculation that involves systematic refinements and matching of the stress distribution at each iteration step. Generalization to the fracture mechanics and other more complicated applications is straightforward since the highstress concentration is a precursor to plasticity and this is exactly indicated by this proposal.

\section{Conclusions}

In this paper, we have analyzed the Kirsch problem of a circular hole in a $2 \mathrm{D}$ material and the generalization including elliptical shapes with different eccentricities, namely the CM and the atomistic-based modelling approaches. We have used a standard description of an ideal graphene sheet with the realistic microscopic interatomic potential and room temperature conditions. We point out that other 2D materials with appropriate interatomic potentials could be treated in a similar way.

The atomistic stress distribution is compared to the continuum limit solution of the benchmark Kirsch problem by examining different conditions and averaging parameters. The differences and the failure of the continuum approach in the area of highest stress concentration were investigated. In order to characterize the loaded system, we reported detailed studies including a variation of the hole geometrical properties. In order to characterize the loaded system, a detailed comparison study of the CM and the atomistic stress distribution was performed. Our quantitative findings clearly show that regions of the $\mathrm{CM} / \mathrm{MD}$ discrepancy are only within the zones of high-stress concentration in the proximity of the very edge of the hole and are realized at the atomic scale.

In conclusion, we also propose simple quantitative criteria for switching from the CM to the MD modelling approach as well as identifying the parts of the model space where the smooth parameterized transition from the CM to the MD modelling is optimal. The main results of this paper are the presentation of the well-known results from CM and a comparison with the MD atomistic description obtained by a suitable averaging procedure. The quantitative comparison factor ( $\mathrm{CM}$ to atomistic ratio) is a basis for detecting suitable locations for the switch between the models during the material loading simulation process.

\section{Acknowledgments}

This work has been fully supported by the Croatian Science Foundation under the project Multiscale Numerical Modeling of Material Deformation Responses from Macro- to Nanolevel (2516).

\section{REFERENCES}

[1] S. Kohlhoff et al.: Crack propagation in bcc crystals studied with a combined finite-element and atomistic model, Phil. Mag. A, 1991, 64, 851-878

[2] E. B. Tadmor et al.: Quasicontinuum analysis fo defects in solids, Phil. Mag. A: Phys. of Cond. Matt., Defects and Mech. Prop., 1996, 73 6, 1529

[3] T. Belytschko, S. P. Xiao: Coupling methods for continuum model with molecular model, International Journal for Multiscale Computational Engineering, 1:12, 2003

[4] B. J. Alder and T. E. Wainwright: "Molecular Motions", Scientific American 201, 113-126 (1959).

[5] M. S. Daw, and M. I. Baskes: Embedded-atom method: Derivation and application to impurities, surfaces, and other defects in metals, Phys. Rev. B, 1984, 29, 6443-6453

[6] L. Verlet: Computer "Experiments" on Classical Fluids. I. Thermodynamical Properties of Lennard, Jones Molecules, Physical Review, 1967, 159(1):98-103,

[7] S. Plimpton: Fast Parallel Algorithms for Short-Range Molecular Dynamics, J Comp Phys 1995, 117 119. https://doi.org/10.1006/jcph.1995.1039, (http://lammps.sandia.gov)

[8] J. Tersoff: Empirical Interatomic Potential for Carbon, with Applications to Amorphous Carbon Phys. Rev. Lett. 1988, 61, 2879. https://doi.org/10.1103/PhysRevLett.61.2879 
[9] J. Tersoff: Empirical interatomic potential for silicon with improved elastic properties, Physical review B., 1988, 38(14):9902-9905

[10] J. C. Maxwell: On reciprocal figures, frames and diagrams of forces. Transl. R. Soc. Edinb. XXVI, 1-43. Maxwell, J.C., 1874. Van der Waals on the continuity of the gaseous and liquid states. Nature 10, 477-480.

[11] J. H. Irving, G. Kirkwood: The statistical mechanics theory of transport processes. iv. The equations of hydrodynamics. J. Chem. Phys., 1950, 18 (06), 817-829.

[12] R. J. Hardy: Formulas for determining local properties in molecular dynamics simulation: shock waves. J. Chem. Phys., 1982, 76 (01), 622-628.

[13] D. H. Tsai: The virial theorem and stress calculation in molecular dynamics. J. Chem. Phys., 1979, 70 (03), 1375-1382.

[14] J. A. Zimmerman, E. B. Webb, J. J. Hoyt, R. E. Jones, P. A. Klein, D. J. Bammann: Calculation of stress in atomistic simulation. Model. Simul. Mater. Sci. Eng. 2004, 12, S319-S332

[15] J. A. Zimmerman, R. E. Jones, J. A. Templeton: A material frame approach for evaluating continuum variables in atomistic simulations, J. Comput. Phys., 2010, 229, 2364-2389. https://doi.org/10.1016/j.jcp.2009.11.039

[16] N. C. Admal, E. B. Tadmor: A unified interpretation of stress in molecular systems. J. Elast., 2010, $100,63-143$.

[17] M. H. Ulz, K. K. Mandadapu, P. Papadopoulos: On the estimation of spatial averaging volume for determining stress using atomistic methods. Modell.Simul. Mater. Sci. Eng. 2013, 21 (1), 15010-15024.

[18] S. Nosé: A unified formulation of the constant temperature molecular-dynamics methods". Journal of Chemical Physics, 1984, 81 (1): 511-519. https://doi.org/10.1063/1.447334.

[19] W. G: Hoover: Canonical dynamics: Equilibrium phase-space distributions, Phys. Rev. A. American Physical Society, 1985, 31 (3): 1695-1697. https://doi.org/10.1103/PhysRevA.31.1695

[20] E. Marenić, A. Ibrahimbegović, J. Sorić, P.-A. Guidault: Homogenized Elastic Properties of Graphene for Small Deformations, Materials 2013, 6(9), 3764-3782, https://doi.org/10.3390/ma6093764

[21] A. Stukowski: Visualization and analysis of atomistic simulation data with OVITO-the Open Visualization Tool, Modelling Simul. Mater. Sci. Eng. 18 (2010), 015012, (http://ovito.org/)

[22] R. C. Sndrew, R. E. Mapasha, A. M. Ukpong, N. Chetty : Mechanical properties of graphene and boronitrene, Physical review B, 2012, 85

[23] E. G. Kirsch: Die Theorie der Elastizität und die Bedürfnisse der Festigkeitslehre, Zeitschrift des Vereines deutscher Ingenieure, 1898, 42:797-807

[24] R. Xu, B. Liu: A Hybrid Molecular Dynamics/Atomic-Scale Finite Element Method for Quasi-Static Atomistic Simulations at Finite Temperature. ASME. J. Appl. Mech. 2013;81(5):051005-051005-7. https://doi.org/10.1115/1.4025807.

[25] E. N. Koukaras, G. Kalosakas, C. Galiotis, K. Papagelis: Phonon properties of graphene derived from molecular dynamics simulations, Scientific Reports 2015, 5, Article number: 12923. https://doi.org/10.1038/srep12923

[26] G. Rajasekaran, R. Kumar, A Parashar: Tersoff potential with improved accuracy for simulating graphene in molecular dynamics environment, Materials Research Express, 2016, 3 (3), 035011. https://doi.org/ 10.1088/2053-1591/3/3/035011

[27] C. Lee, W. Xiaoding, W. K. Jeffrey, H. James: Measurement of the elastic properties and intrinsic strength of monolayer graphene, Science, 2008, 321 (5887), 385-388, https://doi.org/10.1126/science.1157996.

Submitted: $\quad$ 17.7.2017

Accepted: $\quad 12.6 .2018$
Ivan Trapić

Jurica Sorić

Faculty of Mechanical Engineering and

Naval Architecture, University of Zagreb,

Croatia

Robert Pezer

Faculty of Metallurgy, University of

Zagreb, Croatia 